\title{
A scoping review of epidemiological studies of sugar intake in Asian countries: comparison of dietary assessment methodology
}

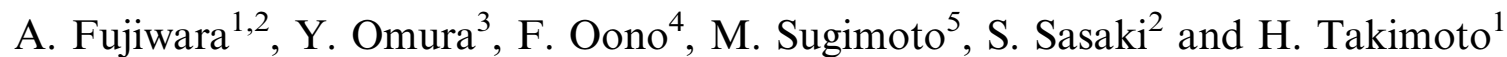 \\ ${ }^{1}$ Department of Nutritional Epidemiology and Shokuiku, National Institute of Biomedical Innovation, Health and \\ Nutrition, Shinjuku-ku, Tokyo, Japan, \\ ${ }^{2}$ Department of Social and Preventive Epidemiology, School of Public Health, University of Tokyo, Bunkyo-ku, \\ Tokyo, Japan, \\ ${ }^{3}$ Department of Internal Medicine, The Jikei University School of Medicine, Minato-ku, Tokyo, Japan, \\ ${ }^{4}$ Department of Social and Preventive Epidemiology, Division of Health Sciences and Nursing, Graduate School of \\ Medicine, University of Tokyo, Bunkyo-ku, Tokyo, Japan and \\ ${ }^{5}$ Institute for Future Initiatives, University of Tokyo, Bunkyo-ku, Tokyo, Japan
}

Several systematic reviews have focused on sugar intake ${ }^{(1,2)}$ and its relationship with health issues ${ }^{(3,4)}$ in mainly Western countries, not Asian counterparts with different dietary habits and disease structures. This scoping review aimed to show the overview of epidemiological studies of sugar intake in Asian countries, primarily focusing on the dietary assessment methodology for estimating sugar intake.

A systematic search was conducted to identify epidemiological studies estimating sugar intake in Asian countries using the PubMed and Web of Science Core Collection on 26 February 2021, with no restriction on publication date. The search strings used were related to sugar, dietary intake, and Asian countries. Inclusion criteria were: published in English; full-text articles in peer-reviewed journals; targeting habitants in Asian countries; estimating self-selected dietary intakes of sugar in participants; and assessing dietary intake using dietary assessment methods at individual level.

Exclusion criteria were: reviews, case reports, proceedings, and abstracts; studies on the contents of sugar in food items or dishes; nonhuman studies; using food supply data; targeting Asian-origin population in Non-Asian countries; using only a biomarker for estimation of sugar intake; estimating dietary intake regulated for the purpose of experiment/treatment; and estimating intake of sugar as foods. Study quality was assessed based on whole diets, dietary assessment methods, and data sources of sugar contents.

In total, 143 studies were identified; 44\% of studies came from Eastern Asia, followed by Southern Asia (21\%), South-eastern Asia $(18 \%)$, and Western Asia (17\%), with no study from Central Asia. More than $65 \%$ of studies investigated total sugars, followed by sucrose, added sugars, fructose, and free sugars (20-15\%). Diet-disease relationships were investigated in $59 \%$ of studies, while $30 \%$ of studies estimated dietary intake and $43 \%$ of studies assessed sugar as the primary exposure/outcome. Regarding dietary assessment methodology, $84 \%$ of studies assessed whole dietary intake, while remaining studies assessed limited food items or occasions. For dietary assessment methods, $43 \%$ of studies used a validated food frequency questionnaire or multiple-day dietary assessment methods. For data sources of sugar contents, $29 \%$ of studies used a comprehensive food composition database developed for individual countries or chemically analysed sugar contents. The remaining studies mainly used a food composition database in other countries or commercial software from other countries. As a result, only $12 \%$ of the total studies and $20 \%$ of the studies with sugar as the primary exposure/outcome were considered to report high-quality data.

This scoping review clarified a sufficient number of epidemiological studies of sugar intake across Asian countries, but most of the studies reported low-quality data. Both feasible and validated dietary assessment methods and comprehensive national sugar composition databases in individual Asian countries are necessary to guarantee the accuracy of estimated sugar intake, and its relationships with health outcomes.

\section{References}

1. Azaïs-Braesco V, Sluik D, Maillot M et al. (2017) Nutr J. 16,6.

2. Newens KJ \& Walton J (2016) J Hum Nutr Diet. 29, 225-240.

3. Moynihan PJ \& Kelly SAM (2014) J Dent Res. 93, 8-18.

4. Te Morenga L, Mallard S \& Mann J (2012) BMJ 346, e 7492. 